\title{
Transposition
}

Musique et Sciences Sociales

Musique : patrimoine immatériel ?

\section{Le jeu du bâton, des pharaons à l'Unesco : patrimoine, identification et construction mémorielle dans le Șa'îd égyptien}

Stick-Fighting Martial Art, from the Pharaohs to UNESCO: Heritage, Identification and Memory Construction in the Sa'id of Egypt

\section{Séverine Gabry-Thienpont}

\section{OpenEdition \\ Journals}

Édition électronique

URL : http://journals.openedition.org/transposition/3133

DOI : 10.4000/transposition.3133

ISSN : 2110-6134

Éditeur

CRAL - Centre de recherche sur les arts et le langage

\section{Référence électronique}

Séverine Gabry-Thienpont, « Le jeu du bâton, des pharaons à l'Unesco : patrimoine, identification et construction mémorielle dans le Sa'īd égyptien », Transposition [En ligne], 8 | 2019, mis en ligne le 15 septembre 2019, consulté le 16 décembre 2020. URL : http://journals.openedition.org/transposition/ 3133 ; DOI : https://doi.org/10.4000/transposition.3133

Ce document a été généré automatiquement le 16 décembre 2020.

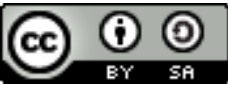

La revue Transposition est mise à disposition selon les termes de la Licence Creative Commons Attribution - Partage dans les Mêmes Conditions 4.0 International. 


\title{
Le jeu du bâton, des pharaons à l'Unesco : patrimoine, identification et construction mémorielle dans le Șa'īd égyptien
}

\author{
Stick-Fighting Martial Art, from the Pharaohs to UNESCO: Heritage, \\ Identification and Memory Construction in the Sa'id of Egypt
}

Séverine Gabry-Thienpont

1 À Louxor et dans ses environs, de nombreux mawālid (fêtes de saints, sg. mūlid) musulmans se tiennent durant le mois de ša ${ }^{\prime} b \bar{a} n$, le mois qui précède celui de ramadān ${ }^{1}$. Chacune de ces fêtes représente une occasion privilégiée pour les hommes du Șa īi - la Haute-Égypte - de se réunir en fin d'après-midi et dans la nuit pour jouer au tahțî̉, le jeu du bâton, également connu sous le nom de la'b al-'așāya. Plus qu'un jeu, le tahțîb a une portée éminemment chorégraphique, ce qui l'amène à être désigné parfois par l'expression raqș al-'așāya, la danse du bâton. La présence indispensable d'instruments de musique entérine l'aspect dansé du jeu : le tahțîb peut difficilement avoir lieu sans mizmār (instrument à anche double de perce conique) et sans țabla baladī (gros tambour à deux membranes). Indissociable de l'identité șa îdī - la pratique comme les musiques ne se transmettraient selon les joueurs qu'entre "fils du Șa îd» (ibn al-Ṣa $\left.a^{i} i d\right)$-, et exclusivement masculin, le tahtîib est un sport empreint de valeurs morales, où l'on fait démonstration de force, de ruse et d'habileté ${ }^{2}$.

Classé depuis novembre 2016 au Patrimoine Culturel Immatériel (PCI) de l'Unesco, le tahtîib constitue un excellent exemple pour questionner la notion de "pharaonisme ». Le pharaonisme (de l'anglais pharaonism) renvoie à une idéologie qui consiste à faire des parallèles entre l'Égypte actuelle et l'Égypte ancienne, arguant l'existence d'une continuité culturelle. Cette manière d'historiciser certaines pratiques conduit à légitimer l'existence d'une identité égyptienne essentialisée. L'histoire admise du tahțiib, qui a contribué à son inscription à l'Unesco, est ainsi avant tout le fruit de spéculations sur sa filiation avec l'Égypte ancienne. Et comme nombre de pratiques 
musicales localement considérées comme ancestrales, le tahtịib est désormais représenté par des joueurs adoubés du statut de "professionnels » dans les qașr altaqāfa (maisons de la culture) du Șa īid sous une forme folklorisée. Pourtant, derrière cette idée de «permanence » qui justifie les entreprises patrimoniales, les pratiques, les normes et les valeurs associées au tahtịib évoluent considérablement, et s'exportent. Étudier les enjeux inhérents à ce jeu permet ainsi d'entrevoir les logiques patrimoniales qui traversent actuellement le paysage culturel égyptien et, en son sein, les musiques égyptiennes, de même que les reconfigurations ultra-contemporaines et transnationales d'un patrimoine estimé ancestral.

3 Partant, les lignes qui suivent proposent une réflexion sur le passage d'une conduite sociale à une pratique culturelle faisant l'objet de politiques patrimoniales définies à des échelles plurielles entre la version ethnographique du jeu et sa (ou ses) version(s) patrimonialisée(s). Dans l'analyse de ce passage, l'accent sera particulièrement mis sur la place de la musique, pour nous permettre de répondre aux questions suivantes : estce que cette place qu'occupe la musique en contexte de performance diffère de celle qu'elle occupe au sein du processus patrimonial? Et au sein de ce processus, quelles sont les composantes estimées indispensables au jeu, pourquoi, et par qui? En m'appuyant sur mes enquêtes ethnographiques, je présenterai d'abord les énoncés du jeu, ses modalités d'exécution et son insertion dans l'espace rural égyptien. J'articulerai ensuite sa mise en forme patrimoniale à un canevas de représentations historiques qui conduisent à redéfinir ce qu'est le tahțîb dans l'Égypte du XXI ${ }^{\mathrm{e}}$ siècle, et, pour certains acteurs, à en proposer une version modelée selon les directives institutionnelles de l'Unesco.

\section{Le tahțīb : du jeu régional au patrimoine national}

4 Abd El Nāser s'avance vers le milieu du cercle formé par une foule d'hommes assis sur de grands tapis en plastique vert et jaune disposés les uns à côté des autres à même le sol, où se mêlent sable et poussière. Nous sommes en 2015, le 20 du mois de ša 'bān, jour de la fête annuelle consacrée au saint musulman Abū Ẓa'būṭ, mort en 1903 et enterré dans le cimetière d'El-Kom³ .

\section{Une pratique précise, des règles strictes}

5 Il fait très chaud. Abd El Naser commence à tourner dans le cercle, frappe le sol de son bâton puis le lève subitement au-dessus de sa tête, fermement maintenu à la base. Abd El Naser est professeur d'arabe dans l'école de Gourna, village voisin d'Al-Ba îrāt. Il se passionne pour le tahțiib depuis son plus jeune âge, jusqu'à être devenu une figure d'excellence dans le jeu. Son père et son grand-père avant lui pratiquaient le tahțîb: Abd El Naser conserve chez lui leurs bâtons en bois épais et lourds, travaillés à l'une de leurs extrémités avec de la peau et ornementés de clous de tapissier. On les désigne par les termes šuba ou nabbūt: c'était l'apanage de "l'homme fort" (fitiwwa; ce mot renvoie à l'idée d'homme fort, protecteur des intérêts locaux, mais aussi d'homme dur, de brute, voire de voyou ${ }^{4}$ ), me dit Abd El Naser. Plus personne n'utilise de tels bâtons dans le jeu : à présent, ils sont faits de bambou (hiżarān), plus léger, et observent une longueur règlementaire (la taille du poing multipliée par neuf). Cela contribue à éviter les incidents graves, comme tuer son adversaire - issue quelque peu regrettable du jeu 
qui se produisait régulièrement il y a quelques dizaines d'années, comme on me le raconte à Al-Ba'īrāt. Mourir au tahțîb est toutefois un acte noble : on m'explique que la famille ne réclame pas vengeance (le ta $a^{\prime} r$, la vendetta) lorsqu'un de ses membres décède des suites d'un coup mortel infligé lors d'une partie de jeu.

6 Abd El Naser dessine le cercle en marchant, le bâton levé, pendant que les zummāra (sg. mizmār) poursuivent leur dōor. Forme musicale tirée d'un poème en dialecte ${ }^{5}$, le dộr stimule le joueur, l'encourage, le retient. Semi-composé, ce dōr montre toute l'importance du rôle du musicien responsable du groupe, le ra'is (chef), qui représente le point de rencontre entre la musique et les joueurs. Des signes furtifs de ces derniers en début de partie, signifiant en un geste quel chant ou quel rythme ils veulent, déterminent le répertoire musical entendu. Le ra'īs doit ensuite être à même d'improviser sur cette base et surtout, il doit faire en sorte que les autres musiciens le suivent. Il est $17 \mathrm{~h}$ : cela fait déjà une heure que le jeu a débuté. Les musiciens ne se sont pas une seule fois interrompus: sur les trois joueurs de zummāra (dits les zammarīn), l'un s'arrête parfois et boit un thé noir brûlant, copieusement sucré, tout en fumant sa cigarette sans filtre "Cleopatra ", laissant les deux autres s'assurer que la mélodie ne s'interrompt jamais. Un adversaire récupère un bâton, s'avance dans le cercle et se positionne face à Abd El Naser. Tous deux commencent alors l'échauffement, en musique, bâton levé, saut sur un pied à reculons, l'autre jambe pliée, pied contre genou. Ils dessinent un cercle. Puis Abd El Naser s'interrompt, s'avance nonchalamment vers les musiciens, et lève la main, index et pouce joints, les trois autres doigts dressés face au ra'iss, signifiant qu'il veut à présent le rythme talat-talata. La partie peut réellement débuter.

7 Tahțiib vient de hațab, le bois sec, référence directe à l'outil du jeu, le bâton. Chaque partie, souvent brève - quelques minutes -, permet à deux joueurs de s'affronter : celui qui gagne est celui qui parvient à toucher le corps de l'autre avec son bâton. Tout l'art de ce jeu réside dans l'enchainement quasi chorégraphique de mouvements et de feintes réalisés avec adresse, précision et en rythme, visant à déjouer les attaques de l'adversaire. Il réside aussi dans l'enchainement de ces courts duels, qui s'étend sur plusieurs heures, voire toute la nuit, au son fort et ininterrompu des mélopées nasillardes des zummāra. Des règles très précises sont respectées, et pas seulement dans le déroulement du jeu : par exemple, le jean, la chemise et le $t$-shirt sont proscrits. Le port de la galabeyya, le vêtement long et couvrant très souvent porté par les hommes de la vallée du Nil, est obligatoire. Un chèche est savamment noué autour de la tête, enserrant bien souvent une calotte de couleur différente. Des deux adversaires, c'est soit le plus âgé, soit l'invité qui commence : il est en effet fréquent, à l'occasion de rassemblements massifs comme lors des mawālid, que des joueurs venus de régions voisines viennent affronter les joueurs locaux. Dans ce cas, la courtoisie du jeu implique de les laisser débuter les attaques. Les étapes sont précises, de même que la terminologie qui s'y rapporte. Chaque partie commence par un salut, signifié par un croisement des bâtons. S'ensuit le ruš, qui renvoie à la manière qu'a le joueur de faire tourner son bâton au-dessus de sa tête en alternance avec son adversaire, tout en marchant à reculons. Puis l'attaque, le pas au rythme de la țabla baladīi le joueur adverse doit la parer au moyen d'al-bāb, la porte. La "porte» est «fermée » quand l'adversaire a bloqué l'attaque en tenant fermement le bâton à l'horizontale entre ses deux mains - le bâton recevant dès lors le coup de l'attaquant. Elle s'« ouvre » quand le joueur ne se protège plus de son bâton et reprend son tournoiement ${ }^{6}$. 


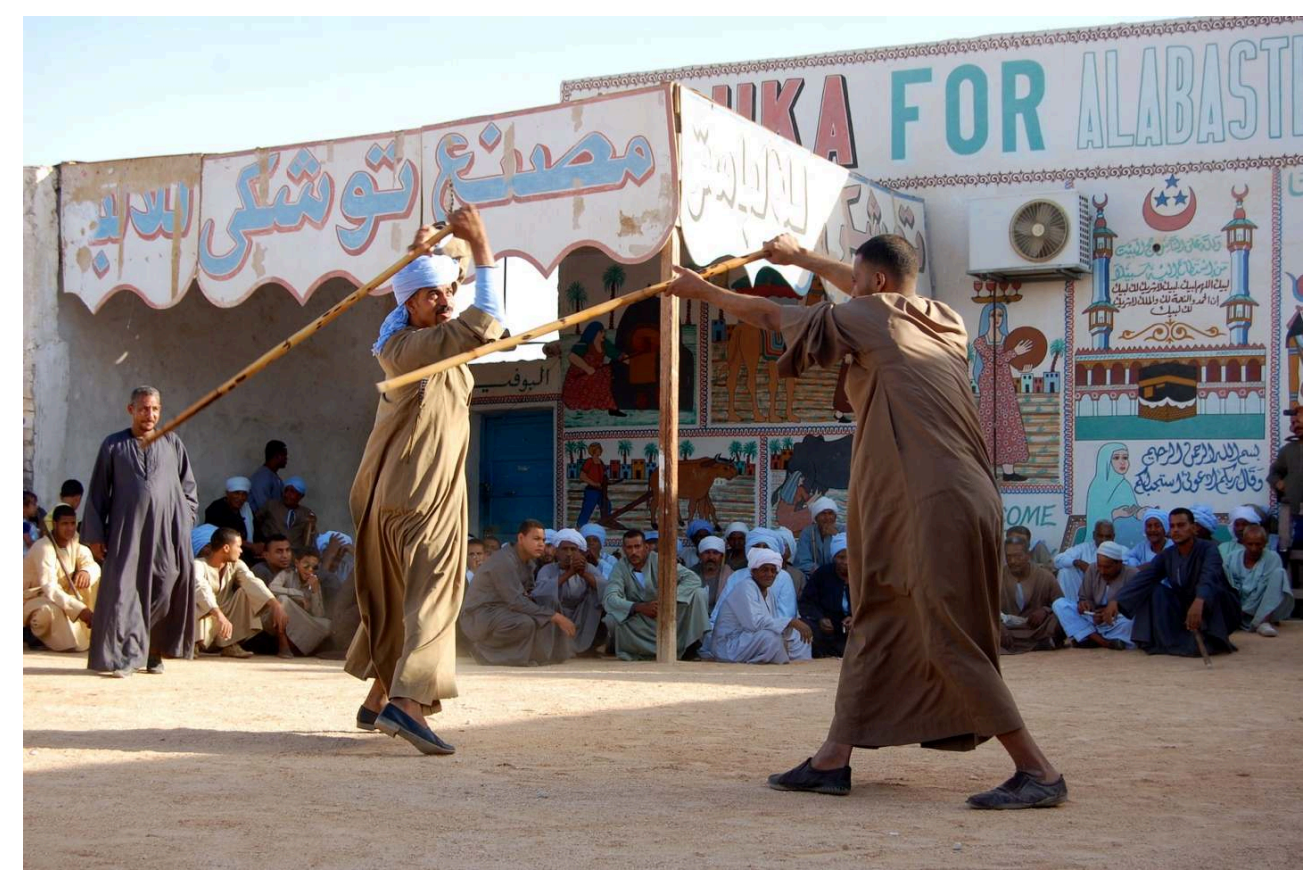

III. 1 : Scène de taḥțīb lors du mawlid dévolu au saint Abū El-Qumșān à Gourna (Haute-Égypte), 2015 (photo de l'auteure).

8 Le tahțīb a un homologue, qui se joue à cheval: le murmāh. Le murmāh est une démonstration de dressage organisé dans le même contexte que le tahțiib, mais plus éloigné du centre nerveux du mouled (associé au tombeau du saint ${ }^{7}$ ). Il s'organise sur de grands terrains en friche réquisitionnés pour l'occasion. Chaque cavalier est déterminé à montrer à la foule et aux autres participants son talent de dresseur, vêtu de sa galabeyya remontée aux genoux, trempé de sueur sous le soleil écrasant, et lançant des interjections plus ou moins violentes à son cheval, le poussant à accélérer, freiner subitement, faire des pas de côté... Tout cela au son lancinant des zummāra et de la țabla baladĩ. Les yeux brûlent, la chaleur, le bruit, la foule étourdissent : l'ambiance a quelque chose d'enivrant. Une quinzaine de cavaliers, parfois plus, participent à chaque murmāh. Tous ont une lance en bois, bien plus grande que le bâton du joueur de tahțîb. Là encore, les règles sont précises, dont certaines - comme celles relatives au contact se rapprochent de celles du tahțib: le jeu est autant une compétition qu'une démonstration de dressage. Le jeu musical diffère légèrement de celui du tahțiib, surtout dans le traitement sonore des anches (les modes musicaux sont les mêmes). Le son du mizmār se fait souvent plus strident, notamment lorsque le musicien souffle tout en frottant rapidement d'avant en arrière les trous de son instrument, provoquant un son qui rappelle le «youyou» des femmes lors des fêtes. Les zummāra stimulent les chevaux, et rappellent au cavalier qu'il ne doit pas oublier de glisser régulièrement un billet lors de ses passages devant les musiciens. Au sein du tahțīb, le jeu musical se fait plus sobre.

\section{La musique comme composante du jeu}

9 Tant dans le tahțīb que dans le murmāh, la musique constitue une composante du jeu indispensable. Ils se sont construits en interaction avec cette musique, et réciproquement : le jeu musical ouvre la séance, juste après la récitation de la fãtih̆a, la première sourate du Coran ${ }^{8}$. L'échauffement des joueurs lui-même, avant de démarrer 
la joute, se fait au son des instruments. Quant à la musique en tant que telle, son jeu est conçu pour soutenir l'action en cours, voire la provoquer: elle n'a pas vocation à se suffire à elle-même et, sortie de son contexte, elle perd son sens et sa fonction. L'un des traits les plus remarquables de cette musique réside dans l'endurance de jeu des musiciens. Cette endurance physique tient à l'apprentissage du souffle continu. Connue aussi sous le nom de respiration circulaire, cette technique est indispensable au jeu de certains instruments de musique, comme la bombarde ou le didgeridoo. Le mizmar est l'un d'eux. Les joues du musicien - forcément un homme - constituent sa réserve d'air, qu'il garde sous pression pour continuer de faire vibrer l'anche double de son mizmar tandis qu'il inspire par le nez. Les musiciens peuvent ainsi jouer pendant plusieurs heures d'affilée, sans s'interrompre pour reprendre leur souffle. Cette question de l'endurance a des répercussions techniques intrinsèques au déroulement tant du tahțiib que du murmāh, puisqu'elle permet le déroulement continu des duels, qui s'enchainent les uns à la suite des autres, et des démonstrations équestres. Elle implique aussi une fragilité accrue de l'anche double du mizmar, qui nécessite pour le musicien de posséder tout un chapelet d'anches de rechange, reliées ensemble et attachées à l'embouchure de l'instrument pour être à portée de main. De cette gestion technique de l'instrument dépend la bonne tenue des joutes. L'aspect structurel du jeu est ainsi doublement lié tant aux contraintes organologiques des instruments qu'à la destination de cette musique, c'est-à-dire au rôle qu'on lui assigne dans le cadre des festivités.

Le tahțîb peut également être accompagné par une rabāba, petite vielle à deux cordes donnant accès au même répertoire, d'un point de vue modal, que le mizmār. Rares sont pourtant les jouteurs intéressés par cet instrument au son moins puissant, donc jugé « moins masculin » de manière explicite par mes interlocuteurs.

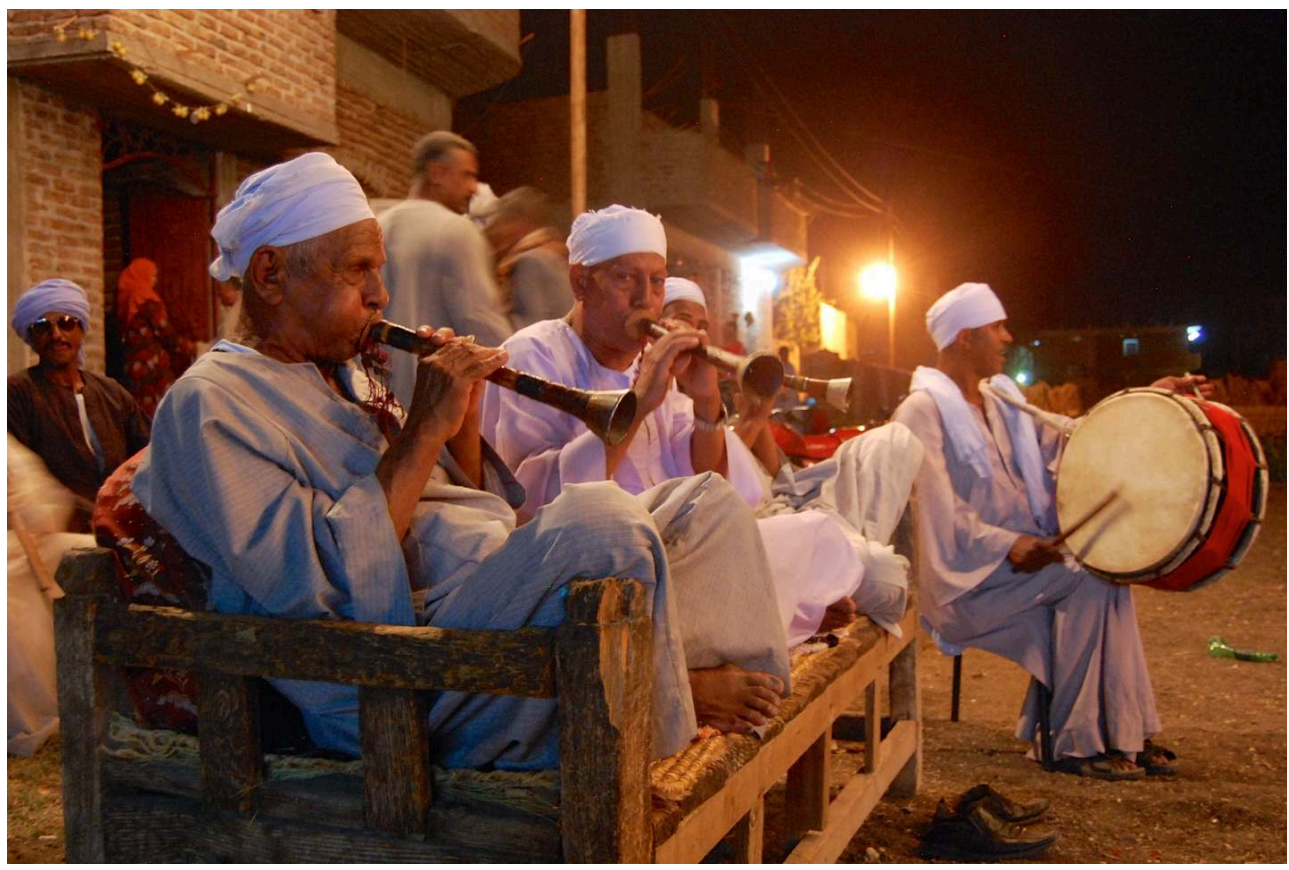

III. 2 : Musiciens jouant mizmār et țabla baladī lors d'une partie nocturne de tahțīb (Al Ba'îrāt, HauteÉgypte, 2015, photo de l'auteure).

11 Au-delà de l'aspect technique du jeu, les joueurs interagissent avec la musique qu'ils entendent, de même que le public: lors du tahtịib, un nouvel arrivant dans le cercle dense des spectateurs est salué par une manière subitement changeante de jouer, ce qui 
a pour sens officiel «Bienvenue à toi!", et pour sens officieux «Nous t'avons remarqué : n'oublie pas de nous payer ». Dans le cas du murmāh, à une première interaction entre les cavaliers, les musiciens et le public, s'ajoute l'interaction avec les chevaux. Les cavaliers paient les musiciens pour participer. Le musicien qui n'est pas payé assez vite le fera savoir par sa manière de jouer, qui correspond aussi à une manière d'exciter le cheval. Ce type de manifestation est tout simplement impossible sans musique. En 2014, lors d'un mouled organisé pour le saint Abu El-Qumșān (Gourna), il s'en est fallu de peu pour que taḥtiib et murmāḥ ne soient annulés : un décès était à déplorer au sein des descendants du saint quelques jours avant la grande nuit, et le deuil ne pouvait être compatible avec le jeu musical des zummāra. Au terme d'âpres négociations, la famille du saint a accepté que les instruments jouent. Soulagement général : « comment aurions-nous fait le tahțīb et le murmāḥ sinon? Ça n'aurait pas été possible !».

Dans un article paru en 2014, l'ethnomusicologue Christine Guillebaud considère une pratique rituelle du Kerala par le prisme de la multimodalité et de l'anthropologie sensorielle. C'est ainsi qu'elle en vient à présenter, au sein de ce rituel, « la relation effective des registres expressifs composant l'action en $\operatorname{cours}^{10} »$. Dans cette réflexion, l'auteure envisage de manière concomitante les « situations d'intersection ${ }^{11}$ » à l'œuvre lorsque plusieurs registres expressifs sont mobilisés - la musique est l'un d'eux. L'exemple est tiré d'un rituel où, par l'usage de poudre colorée, l'élaboration d'un graphisme au sol représentant un entrelacs de serpents s'effectue en musique : « deux médias, à la fois visuel et sonore, sont convoqués au cours de la même action cultuelle ${ }^{12}$ ", action qui s'inscrit elle-même dans un espace spécifique. Dans le cas du tahțiib comme du murmāh, on remarque une imbrication étroite entre performance musicale et bonne tenue du jeu. On remarque également que le choix des instruments est lié à l'espace de jeu, systématiquement vaste et ouvert. Ce choix renseigne sur le rôle de la musique en tant que registre expressif indissociable de l'action en cours : sans musique, l'action n'a pas lieu. Avec d'autres instruments que les zummāra et la tabla baladī, sa mise en œuvre semble également compromise. Il faut de la musique pour lancer les duels, et il faut que cette musique soit d'une forte densité sonore pour contribuer pleinement à créer l'atmosphère recherchée, constitutive du jeu, mais aussi de l'idéal de masculinité qu'il supporte.

\section{Un idéal masculin qui mène à la reconnaissance locale d'un patrimoine}

13 En Égypte, le tahțiib est représenté au sein des qașr al-țaqāfa, " maisons de la culture » qui dépendent du ministère de la Culture. Ces établissements permettent à certains musiciens d'accéder au statut envié de fonctionnaire, redéfinissant dès lors les réseaux de relation entre joueurs et musiciens, leur notoriété et les contextes de performance. Le tahtîib a acquis une visibilité au sein de l'État, qui autorise l'organisation de compétitions interrégionales, en collaboration avec le ministère des Sports. En lien avec la programmation de ces maisons de la culture, des "équipes» régionales de joueurs se réunissent régulièrement dans une salle prévue à cet effet pour se préparer à ces rencontres. Dans ce cas en particulier, les passes du tahtịib sont hiérarchisées selon un décompte de points. "Toucher" la tête de l'adversaire permet de remporter 45 points; le bras, 8 ; réussir à fermer la bāb à temps en donne 32 . Ce système de points 
n'est valable que dans ces cadres officiels: dans les mawālid, les fêtes de mariage ou autres festivités privées, cela n'entre nullement en ligne de compte. Toutefois, cela a un impact sur le statut des joueurs: les meilleurs joueurs que l'on croise dans diverses régions et qui prennent plaisir à se déplacer parfois loin pour jouer lors d'un mūlid particulier, ont pour la plupart déjà tenté leur chance lors des compétitions officielles, organisées par l'État. Abd El Naser a déjà remporté deux médailles. Il est considéré comme l'une des principales figures du tahțîb de la région de Louxor.

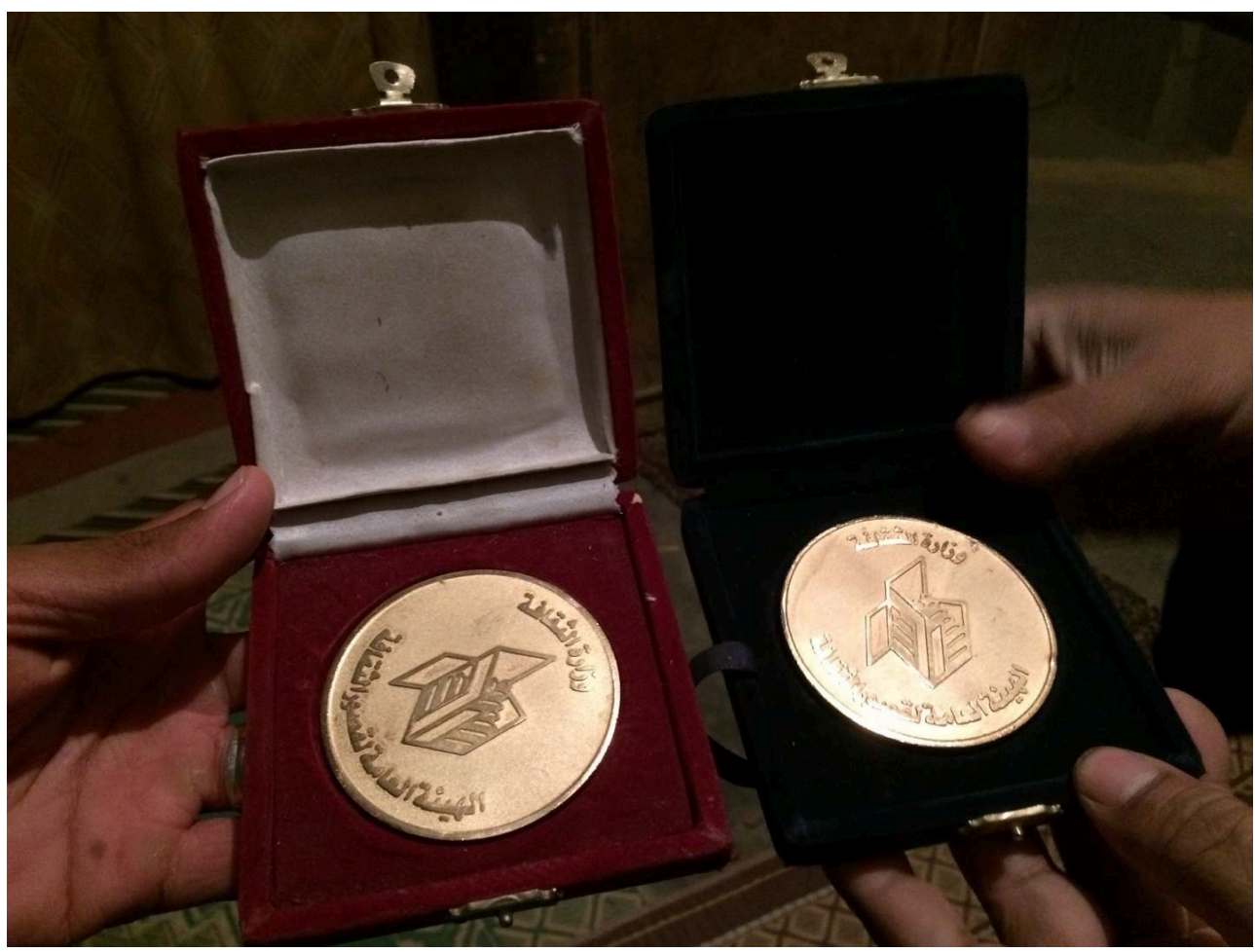

III. 3 : Médailles remportées par Abd El Naser, délivrées par le ministère de la Culture.

En proposant d'ouvrir le tahțiib à la compétition officielle, l'État égyptien l'a entériné comme pratique culturelle légitime : il l'a doté d'une crédibilité et reconnaît l'existence de bons joueurs. Ces derniers, comme les représentants officiels, désignent le tahțib comme du turat al-taqā $\bar{i}$, du patrimoine culturel ${ }^{13}$. La racine trinitaire du mot turat, $w-r-$ t, renvoie à la fois au fait d'hériter de quelque chose et d'être l'héritier de quelqu'un. Mais cette racine renferme aussi l'idée d'hérédité. Par son étymologie même, ce mot renvoie donc à une notion de transmission d'un héritage ou d'un patrimoine, quelle qu'en soit la nature. Avec cette reconnaissance en tant que "patrimoine culturel», le tahțiib s'affiche comme une pratique ancestrale qui serait préservée particulièrement en Haute-Égypte. Pourquoi une telle considération étatique?

Le tahțiib est considéré comme porteur de valeurs morales et physiques qui correspondent à un idéal national égyptien, idéal lui-même associé, en réalité, au fait d'entériner une partition genrée de la société. Mais cet idéal est surtout véhiculé dans le sud du pays. En 2013, le spectacle de fin d'année de l'école publique de Gourna, village de Haute-Égypte où vit Abd El Naser, donnait à voir une chorégraphie réalisée par les garçons de l'école. Vêtus d'une galabeyya de couleur claire, un chèche noué autour de la tête et un bâton dans une main, ces garçons dansaient au son de la chanson célèbre Eh Ya Lalaly, interprétée par le groupe égyptien soft rock Wust el Balad. Dans cette chorégraphie, les garçons alternaient des gestes associés aux règles du jeu du 
tahțiib, comme la position de l'échauffement - saut sur un pied, bâton levé - et des enchainements purement chorégraphiques, tels des rondes ou des sauts alternés sur un pied, genou levé, comme on pouvait en voir dès les années 1950 avec la création en Égypte de la troupe de danse des frères Reda, qui a accordé une place de choix au tahțiib dans ses compositions chorégraphiques. Cette danse de spectacle était uniquement masculine, à l'exception de l'entrée discrète d'une jeune fille, autour de laquelle dansèrent un bref instant les garçons. Les familles présentes dans l'assemblée frappaient des mains au rythme de la musique, le visage fendu d'un sourire amusé, mêlé de fierté : ces garçons, non moins fiers de porter les attributs de jeu du tahtîb, illustraient par cette danse une représentation socialement héritée du Șa î̀ī, l'homme du Șa īid.

Le tahțib s'illustre en effet par une certaine version de la masculinité, qui passe tant par le jeu que par la danse. Un lien étroit est installé entre les composantes du tahțiib et cette masculinité du Șa'id, magnifiant une "identité sa'idi ", de la même manière que le note l'anthropologue Catherine Miller au sujet du dialecte sa'idi, différent en bien des points du dialecte cairote : lorsqu'un homme originaire de Haute-Égypte s'installe au Caire, qu'il perd son accent et ses habitudes dialectales, et qu'il revient après un certain temps dans son lieu d'origine, ses proches attendent de lui qu'ils reprennent son dialecte local, sans quoi il est traité de fäfi, littéralement, de quelqu'un d'efféminé ${ }^{14}$. Pratiquer le tahțiib, et le pratiquer brillamment, rappelle cette considération du dialecte du sud : cela signifie qu'on est un homme, un vrai. Pourquoi ? D'abord, le tahțîb, dans sa forme ethnographique décrite plus haut, véhicule une certaine conception de la masculinité propre à la société du sud de l'Égypte. Cette masculinité passe par des attributs concrets, par exemple les normes vestimentaires, qui touchent aussi bien les joueurs que les musiciens. L'homme doit en effet porter une galabeyya, on l'a vu. Celleci doit être propre, bien repassée, et accompagnée de mocassins aux pieds ou, à défaut, de toute autre chaussure fermée. Il est impensable pour un joueur de porter des chipchip - référence au bruit que font leurs propriétaires en marchant-, ces sandales en plastique bon marché qu'on trouve au souk et que chacun, homme, femme, enfant, porte au quotidien dans le Șa î̀ : il préférera, dans ces cas-là, y aller pieds nus. La consommation constitue un deuxième exemple. Elle reflète une sociabilité masculine : bière et cannabis font légion, de même que les cigarettes, véritable relai social, puisque les hommes s'en offrent mutuellement au sein du cercle des spectateurs, entamant ainsi maintes conversations. Si le tahțib se déroule lors d'un mawlid près du tombeau du saint, il ne sera pas question de consommer ouvertement de l'alcool. La tolérance est plus étendue en ce qui concerne la consommation de cannabis. Pour une femme, en revanche, il n'est pas convenable de consommer ces produits, surtout dans des espaces publics. La musique, enfin, représente un troisième exemple : seuls les hommes jouent des zummāra et de la țabla baladī, on l'a vu - «beaucoup trop compliqué pour une femme », m'a dit un jour sur un ton d'évidence un musicien interrogé. Quant au son, il doit être fort et puissant, d'où l'usage privilégié du mizmār : à travers l'expression musicale se dessine ainsi toute une conception de la masculinité.

17 Mais de quelle masculinité parle-t-on? Dans son ouvrage Masculinities ${ }^{15}$, la sociologue Raewyn Connell propose le concept de "masculinité hégémonique », théorisant une forme de domination masculine qu'elle identifie comme une perpétuation du patriarcat dans les rapports genrés aussi bien féminins que masculins - le cadre théorique propose en effet une distinction des «masculinités» existantes. Affaire d'hommes gérée par les hommes, le tahțiib représente la légitimité du patriarcat, donc d'une 
domination masculine, " hégémonique ", s'exerçant socialement au vu et au su de tous. Cette domination s'exerce à travers la manifestation de la force des joueurs, une force qui dépasse éminemment la considération simplement physique des individus : elle s'exprime autant à travers l'atmosphère et la puissance sonore du jeu musical, qu'à travers la ruse, l'habileté, l'honneur et la défense des siens, tant de qualités socialement considérées comme des vertus masculines. C'est l'association de ces qualités qui permettent aux grands joueurs de se reconnaître, voire d'asseoir leur supériorité sur d'autres - certains joueurs sont en effet localement très célèbres, et affublés de surnoms en lien avec ces valeurs masculines ${ }^{16}$.

Cette domination masculine s'exerce également dans l'assemblée des spectateurs et dans la gestion de l'espace : les zones de jeu sont ouvertes et, de préférence, vastes des champs en friche, des terrains vagues, de larges rues -, les démonstrations sont publiques. Ces rassemblements masculins se différencient des rassemblements féminins, qui impliquent un entre-soi, une intimité, des espaces clos et confinés, à l'abri des regards. Le tahțiib oppose une masculinité empreinte de virilité et de démonstration de force, "valeurs masculines" que les hommes proposent très tôt aux enfants comme on l'a vu avec la fête de l'école de Gourna-, à une pudeur et une réserve socialement perçues comme des "valeurs féminines». Dans certains articles de journaux égyptiens consacrés au tahțîb, on lit parfois que ce jeu serait une expression de séduction à destination de la gent féminine. L'idée est vite contredite par l'absence effective des femmes dans les espaces de jeu. De petits groupes se forment parfois pour assister aux parties, mais ils restent discrètement à l'écart et préfèrent les parties nocturnes, où leur présence sera moins visible qu'en plein jour. Le tahțīb invite à examiner un rapport de force qui s'établit avant tout entre hommes.

Le jeu du bâton, avec sa musique, donne ainsi à observer l'élaboration d'une masculinité spécifique, en adéquation avec l'idéal masculin tel que défini par les normes sociales du Șa îid. Cette conception de la masculinité s'exerce dans un rapport d'inclusion : une communauté d'hommes se construit autour de la pratique musicale et chorégraphique du tahțiib, tissage singulier constitué de jeu, de danse, de musique et de codes moraux. Ce faisant, cette mise en place communautaire donne corps à un idéal de masculinité, au moyen de plusieurs registres expressifs. Le jeu, avec sa musique, en devient représentatif d'une forme dominante et largement répandue de ce qu'est la «masculinité » de Haute-Égypte, que nous prenons le parti de nommer «masculinité șa idī ». En s'avérant socialement et culturellement recevable, ce jeu pouvait s'inscrire de manière légitime comme du turat (patrimoine) et obtenir cette qualification dans les maisons de la culture égyptiennes.

\section{L'histoire admise du tahțīb et son inscription au PCI}

Le bâton du Tahtib est porteur du respect, de la dignité, de l'engagement à s'accomplir dans son humanité. L'art du Tahtib remet le bâton à sa place, tel que représenté sur les fresques anciennes : celle du compagnon de route, celle du lien à la terre, celle de la protection d'autrui, celle de la parole et du sens et celle de la fête partagée avec les autres. La contribution de l'art martial 
égyptien EL TAHTIB est double, dans le monde des arts martiaux, avec son souffle remarquablement festif et participatif, en Égypte, comme vecteur éducatif et d'émancipation. L'art du bâton a traversé plus de 5000 ans $^{17}$.

20 Au-delà de la question de l'idéal masculin, qui permet au jeu du bâton d'être culturellement légitime en Égypte, le phénomène de reconnaissance du tahtịib en tant que patrimoine est à mettre en lien avec un certain rapport au passé. Il « offre aux acteurs l'opportunité d'une nouvelle réappropriation et d'une nouvelle conscience historique, confrontés qu'ils sont à de multiples passés que la patrimonialisation rend plus présents ${ }^{18} »$.

\section{«Pharaonisme » et continuité culturelle}

21 En 2014, quand je projetais de débuter mes enquêtes sur le tahțib, je venais d'être recrutée à l'Institut français d'archéologie orientale du Caire (Ifao). L'Ifao est bien connu du monde des archéologues et des historiens de l'Antiquité, en tant que fief des études égyptologiques depuis le XIX ${ }^{\mathrm{e}}$ siècle. J'y ai rencontré l'égyptologue Sibylle Emerit, spécialiste de la musique et de la danse. En 2013, cette chercheuse avait été contactée par Adel Boulad qui cherchait à obtenir des preuves égyptologiquement recevables de la continuité dans le temps et l'espace du jeu du bâton et de sa musique. À partir d'une hypothèse fondée sur l'analogie des formes, il était par exemple convaincu - à tort selon S. Emerit - que la trompette égyptienne ancienne représentée sur les parois des temples du Nouvel Empire, était en réalité un mizmār-notons que le contexte pariétal en question n'avait pas de rapport avec le jeu du bâton. À cette époque, Adel Boulad souhaitait faire inscrire le jeu du bâton au Patrimoine culturel Immatériel de l'Unesco - ce qui est chose faite depuis novembre 2016 - et avait besoin, pour cela, de renseignements précis quant à l'histoire du tahțiib. Cet amoureux du jeu s'était rapproché de l'Ifao pour mêler les enjeux esthétiques du tahțî̀ à une approche historique et scientifique. Selon une préconception tenace, le tahtîib est en effet massivement considéré comme l'héritier d'une pratique masculine recensée sur des représentations pariétales, datant de près de 5000 ans. Cette continuité supposée n'a jamais été solidement argumentée, mais c'est ainsi qu'il est présenté dans les articles de journaux égyptiens et que, dans leurs discours, Abd El Naser et les autres joueurs évoquent ce jeu. En proposant une lecture qui se veut historique du tahțîb, ces acteurs cherchent à en authentifier la pratique et à lui donner une consistance durable. L'histoire du tahțiib répandue de nos jours est donc que cette pratique aurait traversé les siècles, et même, les millénaires, transmis dans un souci de fidélité par des générations d'hommes. Partant de ce postulat, seul le recours à l'égyptologie semblait légitime à Adel Boulad, comme à d'autres, pour retracer l'histoire de l'origine du tahtịib et de sa musique.

En Égypte, le tahțiib n'est pas la seule pratique qui bénéficie de tels discours d'authenticité plurimillénaire. Le travail que je mène là-bas en tant qu'ethnomusicologue depuis maintenant dix ans me fait entendre nombre de discours autour de la continuité culturelle de l'Égypte et de ses manifestations. C'est le cas au sein des pratiques chantées coptes : il est en effet considéré comme acquis aussi bien par les savants occidentaux qui se sont intéressés à ces chants tout au long du 
$\mathrm{XX}^{\mathrm{e}}$ siècle, que par bon nombre de coptes et de musulmans, que ces chants sont les héritiers des musiques de l'Égypte ancienne, et qu'ils auraient une persistance durable $\mathrm{du}$ fait du conservatisme de l'Église copte d'Égypte ${ }^{19}$. Dans la mesure où il ne convient pas de changer ce que les coptes ont reçu de leurs Pères, et que le christianisme a succédé au paganisme avant l'arrivée de l'Islam au VII ${ }^{e}$ siècle, l'Église aurait conservé l'antique musique de ses illustres ancêtres. Non seulement cette assertion est largement diffusée, mais elle reçoit de plus une caution scientifique de chercheurs désireux de prouver l'égyptianité de cette Église ${ }^{20}$. Ces discours ont émergé à une époque propice aux discours nationalistes, et l'Église copte, en rattachant son existence à un passé prestigieux d'avant la conquête arabe, valorisait par là même une identité strictement égyptienne, pour mieux contourner un statut inconfortable de minorité. Le fantasme d'associer les chants coptes à l'Égypte ancienne reste solidement ancré, et fait même partie de l'image que d'aucuns ont de ces chants, en dépit de toute étude scientifique qui permettrait de retracer l'histoire sonore et musicale de l'Église copte.

Ces mécanismes de réappropriation historique et ce goût pour les origines prétendument antiques des pratiques sont déjà décrits dès les années 1980 par Edward Said, dans son incontournable L'orientalisme : l'orient créé par l'occident. Dans cet ouvrage, E. Said définit l'orientalisme comme un "style de pensée fondé sur la distinction ontologique et épistémologique entre "l'Orient" et (le plus souvent) "l'Occident" 21 ". C'est effectivement dans une mouvance orientaliste - que l'on peut qualifier d'idéologique - que de nombreux savants et chercheurs européens au XIX ${ }^{\mathrm{e}}$ et $\mathrm{XX}^{\mathrm{e}}$ siècle ont élaboré leurs propos et fondé leurs hypothèses. À partir de cette "distinction ontologique ", nombre de théories autour de la transmission et de l'histoire des pratiques égyptiennes ont été composées. Elles ont été revendiquées comme très anciennes, la plupart du temps dans l'idée d'interpréter des scènes antiques à partir de constats contemporains, donnant alors naissance à de nombreuses préconceptions ${ }^{22}$. Un autre exemple frappant est celui d'un pèlerinage dévolu au saint patron de la ville de Louxor, le saint Abu El-Ḥağğağ, qui vécut au XIII ${ }^{e}$ siècle. Les festivités organisées pendant une semaine durant le mois qui précède ramadan en l'honneur de ce saint soufi attire des foules de visiteurs. Au terme de cette semaine se tient la dōra (la procession), marquée par un défilé de micro-bus, de chameaux, mais aussi de barques chargées de personnes et munies d'enceintes tonitruantes. Comme Rachida Chih l'a noté dans un article paru en 1993, "certains chercheurs occidentaux ont pendant longtemps interprété les rites et traditions populaires des Égyptiens comme la survivance de rites pharaoniques et chrétiens ${ }^{23}$ ». Parmi eux, R. Chih cite l'égyptologue français Georges Legrain, qui écrivait en 1914 :

Il est rare qu'un Européen puisse pénétrer dans la mosquée d'Abû-l-Hajjâj, en visiter les tombeaux et voir la barque du saint. Car celui-ci, ayant supplanté Amon en se logeant dans une partie de son temple, a hérité ses mérites fécondateurs, ses charges et ses attributs. Et, comme Amon jadis, Abû-l-Hajjâj fait une fois l'an, en barque, le tour de sa bonne ville de Louqsor... Au moment de la procession, la barque est chargée sur un support à quatre roues et recouverte de la grande étoffe multicolore qui, pendant l'année, dissimule la tombe du saint. Remorquée par les fidèles, ornée de drapeaux, la barque du saint avance lentement au milieu des chants, des acclamations, des prières et des coups de feu de la foule en délire qui se masse pour toucher le voile du saint, sous lequel sont accroupis quelques enfants privilégiés ${ }^{24} .$. barque associée à Abū El-Ḥağğăğ, mais il est manifeste que, comme pour les chants 
coptes, une telle analogie avec des rites anciens a d'abord été le fruit de spéculations de savants orientalistes, désireux de croire qu'il était possible de prouver une survivance tangible de pratiques antiques. L'idée était encore présentée telle quelle dans un film documentaire consacré à ce pèlerinage (réalisé par Alain Bellod en 1982 ${ }^{25}$ ). Dès les premières minutes, une introduction est proposée par l'égyptologue Claude Traunecker qui montre des scènes antiques de procession : ses premiers mots laissent entendre ce désir de donner vie à ces représentations, et il indique ainsi ce lien entre les processions consacrées aux fêtes du dieu égyptien Opet et celles dévolues à Abū ElḤaǧğāă : «Ils [les Égyptiens de Louxor] recréent les anciennes fêtes pharaoniques, ce qui est quelque chose d'assez puissant ", manifestant la charge émotionnelle que peut représenter la faculté à toucher du doigt le passé. Faire des ponts avec l'Antiquité a donc été une idée qui a dominé tant l'orientalisme du XIX ${ }^{e}$ siècle que celui du $\mathrm{XX}^{\mathrm{e}}$ siècle, et qui a interféré au sein de nombreuses approches scientifiques. Dans un même processus de "métamorphose» de l'orientalisme et de phénomènes d'appropriation locale au cours du XX $\mathrm{X}^{\mathrm{e}}$ siècle, présentés par F. Pouillon et J.-C. Vatin ${ }^{26}$, les joueurs de tahțiib revendiquent à présent la haute antiquité et la fiabilité de la transmission de leur pratique : la considération d'une continuité culturelle intangible n'est plus l'apanage d'observateurs extérieurs. L'aspect «ancestral»- mawrūt, qui porte la même racine arabe que le mot turat, patrimoine - de ce jeu en fait l'une de ses composantes à part entière : que le tahțiib soit du turat participe désormais de sa nature en tant que pratique mawrūta $a$ (ancestrale), et entérine sa légitimité territoriale.

Or cette projection du passé dans le présent dessine l'histoire en cours de cette pratique. Mercedes Volait, spécialiste de l'histoire architecturale et patrimoniale de l'Égypte moderne, a travaillé sur l'orientalisme architectural du XIX siècle, qui puise bien souvent dans le registre du style néo-classique. Elle évoque dans ses travaux la profusion de productions architecturales exotiques reproduisant ou fantasmant des données historiques, en revendiquant leur qualité de "savoir construit ${ }^{27}$ ». Dans leur introduction à L'Orientalisme architectural entre imaginaires et savoirs, M. Volait et N. Oulebsir expliquent cette notion de "savoir construit » en se référant aux sources d'inspiration en jeu, puisées dans l'Égypte ancienne, et à ce qu'elles renseignent sur les conceptions d'altérité et de représentation de soi. Elles incluent également la théorisation artistique qui découle de ces productions exotiques et la sélection de modèles qu'elles impliquent. Le tahțīb, par son histoire récemment admise et diffusée, témoigne lui aussi d'un "savoir construit». Considérer cette construction permet d'analyser les enjeux de la considération du jeu du bâton en tant que patrimoine, tout comme les désirs tant étatiques que privés de promouvoir un art désormais revendiqué comme national. Plus généralement, analyser cette construction renseigne sur l'esthétique qui se dessine derrière ces pratiques populaires, une esthétique qui a évolué au fil de l'histoire, oscillant entre mépris et valorisation de la part des Égyptiens en général. Cette esthétique est désormais imprégnée d'une volonté d'égyptianiser ce jeu, en le rattachant à un passé glorieux, teinté d'un fantasme de perfection. Localement formalisés et banalisés, ces discours alimentent ainsi une histoire des pratiques parfois partisane à défaut d'être suffisamment étayée par de solides justifications scientifiques. Cette histoire, certes difficile à justifier, nous renseigne néanmoins sur tout un pan de l'histoire culturelle de l'Égypte contemporaine, sur son esthétique et sur les motivations qui gravitent autour. 


\section{" Modern Tahtib » et PCl}

novembre 2016, le tahtīb est inscrit sur les listes du Patrimoine Culturel Immatérie de l'Unesco, grâce à l'action de Adel Boulad. Issu d'une famille syro-libanaise, Adel Boulad est né en Égypte en 1951. Passionné d'arts martiaux, il s'intéresse au tahțiib dès les années 2000 et se lance en 2013 dans la procédure d'inscription du tahțîb au PCI. Dans la dynamique de reconstitution historique précédemment présentée, A. Boulad a constitué une certaine histoire du tahțỉb, qu'il présente à l'envi lors d'interviews télévisées et écrites. La frise chronologique qu'il suit est la suivante : il évoque une histoire de trois mille ans d'art martial et militaire, durant laquelle l'Égypte pharaonique aurait utilisé le tahțīb comme art de guerre. Il parle ensuite de deux mille ans de jeu « rural ", puis de cinquante à soixante-dix ans de jeu «folklorique » ${ }^{28}$. Dans sa conception du jeu, Adel Boulad différencie pratique "rurale» et pratique "folklorique ", cette dernière étant sans doute une référence à la manière dont les frères Reda ont donné à voir le tahțīb au sein de ses chorégraphies d'inspiration «traditionnelle ». Il se peut qu'A. Boulad voie la manière de faire aujourd'hui le tahtịib comme influencée par le développement folklorique qu'en a proposé cette troupe ${ }^{29}$.

Pour être inscrit au PCI de l'Unesco, et être donc considéré institutionnellement comme $\mathrm{du}$ patrimoine, plusieurs critères ont dû être remplis. Voici quelques extraits du procès-verbal de 2016 rendant officielle l'inscription du tahțî̉, consultable en ligne sur le site de l'Unesco :

L'État soumissionnaire démontre que le tahteeb constitue un élément du patrimoine culturel immatériel tel que défini à l'article 2 de la Convention. Les communautés, groupes et individus concernés, répartis dans la quasi-totalité du pays, considèrent qu'il fait partie intégrante de leur patrimoine culturel et promeut le respect mutuel, la dignité et la cohésion sociale, procurant ainsi un sentiment d'appartenance à ses praticiens. [...]

Le tahteeb est un élément du patrimoine immatériel profondément enraciné dans la société égyptienne; ses origines remontent à l'Égypte ancienne; sa forte présence dans la culture égyptienne a été perpétuée à travers les différentes ères de son histoire jusqu'à maintenant dans la mesure où ses traditions et règles reflètent les valeurs essentielles de la société telles que la fierté, la chevalerie, le courage et le respect. [...]

L'État soumissionnaire décrit des mesures de sauvegarde qui incluent la participation active des communautés concernées à leur élaboration et à leur mise en œuvre, notamment à travers le programme de formation des formateurs (PFF) pour préparer les nouveaux formateurs et pratiquants du tahteeb ${ }^{30}$.

Pour être en mesure de remplir ces critères, Adel Boulad a constitué au préalable un dossier - réalisé notamment en collaboration avec Ayman Khoury ${ }^{31}$ - qui a nécessité de parcourir la Haute-Égypte pour recueillir entretiens et témoignages, ainsi que des images de la pratique (photos et séquences filmées). Il a également fallu obtenir la signature du ministère égyptien de la Culture. Mais, surtout, Adel Boulad a créé le Modern Tahtib 32 .

A. Boulad présente son Modern Tahtib comme un art martial inspiré du tahțiib «méthode actualisée du combat au bâton égyptien », peut-on lire sur le site officiel. Inauguré en 2013, ce projet était en gestation dès le début des années 2000 au sein de l'association Seiza. Créée en 1978, Seiza est affiliée à la Fédération française de Karaté et Arts martiaux. C'est une association transdisciplinaire, où se pratique tout type d'art martial japonais. Au début des années 2000, l'association commence à organiser des 
stages entre divers clubs, pour que ses adhérents s'initient au maniement du bâton au contact d'arts martiaux dont la pratique est en lien avec ce genre d'arme. Puis, en 2003, un stage est organisé avec Mohamed El Sayed, danseur et musicien égyptien, fin connaisseur du tahtiib. Cette pratique s'immisce ainsi progressivement dans le paysage sportif des adhérents.

30 A. Boulad conçoit le Modern Tahtib comme un développement contemporain du tahțīb. En le sortant des frontières égyptiennes, il tente d'établir le tahțîb « dans sa vocation universelle comme levier sociétal et culturel de développement de la personne et du collectif, avec une base robuste et pérenne en France $^{33}$ ». Comme en témoigne le troisième extrait du procès-verbal suscité, l'inscription au PCI implique en effet d'assurer une transmission de la pratique ${ }^{34}$. A. Boulad a ainsi mis en place un programme de formation d'instructeurs, dont toutes les règles sont explicitées au sein de l'ouvrage Modern Tahtib, bâton de combat égyptien, paru en 2014. Cette transmission doit constituer, dans le cadre de l'inscription à l'Unesco, un moyen de pérenniser ce jeu, d'envisager que ce patrimoine continue à se développer, mais aussi, en filigrane, d'épurer la pratique locale de son apparat problématique : le Modern Tahtib est ainsi ouvert à tous, femmes comprises. Boulad voue également cet art martial à devenir une source d'inspiration pour les futurs "dirigeants ", comme il les nomme, en proposant des interventions au sein des grandes écoles et d'universités prestigieuses ${ }^{35}$. Il a ainsi proposé un stage d'initiation au sein de l'association sportive de Sciences Po Paris en 2014, accompagné d'une darboukka ${ }^{36}$, ce qui a suscité nombre de réactions enthousiastes de la part des élèves.

31 L'élaboration de cet art martial tient tant du tahțiib tel que les Égyptiens le pratiquent aujourd'hui dans le sud du pays, que de son histoire et des nombreux discours que je mentionnais précédemment sur son "égyptianité ». Conçu comme un art martial, le Modern Tahtib assume sa position novatrice, sans se revendiquer de ce qui voudrait être perçu comme une sorte de "vrai » tahțiib, de pratique pseudo-authentique qui aurait traversé les âges sans jamais subir la moindre altération. Dans l'ouvrage paru en 2014, les justifications historiques sont toutefois parties prenantes de l'explication de ce qu'est la discipline. Elles font même l'objet d'un chapitre à part entière ${ }^{37}$. On peut également y lire des explications philosophiques. Ainsi, durant ses cours, A. Boulad développe une approche à la fois philosophique et spirituelle (approche constitutive de tout art martial) qui s'appuie sur une interprétation des images pariétales de l'Égypte ancienne. Selon cette approche, et concernant plus particulièrement la musique pratiquée lors du tahțiib, le son aigu du mizmar apporte la dimension haute du tahțib, c'est-à-dire le Ciel. Le son des percussions représente quant à lui la dimension basse : la Terre. « Le son du mizmar produit un effet d'élévation et celui des percussions, un effet d'ancrage avec la Terre sur les pratiquants du tahțï $b^{38} »$. A. Boulad ajoute que «ce lien entre Ciel et Terre est symbolisé dans l'art égyptien de différentes manières et fait partie des bases spirituelles de l'Égypte des Pharaons ${ }^{39}{ }^{\prime}$. Toutefois, dans ce Modern Tahtib, il est rare qu'une place soit accordée à la musique, généralement pour des raisons de faisabilité. Les représentations officielles lors de compétitions s'accompagnent parfois de percussions, sans que cela soit présenté comme une condition sine qua non à la bonne tenue du jeu, et aucun instrument mélodique de type mizmar n'est intégré. La musique, élément indispensable dans le jeu en Haute-Égypte, ne semble pas être considérée comme une composante nécessaire au point de devoir l'intégrer dans la réalisation d'un développement moderne du tahțiib. 

au PCI, en impliquant un développement au-delà de la Haute-Égypte et pour tous, hommes et femmes, «sous une forme structurée, codifiée, sportive et pacifique ${ }^{40}$ ». Il est intéressant de remarquer que l'une des différences fondamentales est la quasiabsence de musique dans le Modern Tahtib, ou, éventuellement, juste quelques percussions. La conception multimodale, pour reprendre l'expression de Christine Guillebaud, ou la considération des différentes modalités ou composantes de cette pratique telle qu'inscrite au PCI, ne constitue pas une constante. Il n'y a pas d'interactions, donc pas d'approche intégrative des différents modes d'expression tels qu'ils existent actuellement dans cette pratique en Haute-Égypte : la différence majeure entre ces deux pratiques réside précisément dans cette place qu'on accorde - ou pas - à la musique.

\section{Conclusion}

À l'aide ce cette présentation de la pratique du tahțỉb dans l'Égypte du XXI ${ }^{e}$ siècle et des récents développements qui ont mené à la création d'un Modern Tahtib par Adel Boulad - qui a amplement contribué à l'inscription à l'Unesco -, l'analyse visait à montrer comment et pourquoi le tahțīb a été érigé en pratique "ancestrale», d'abord localement, et comment cet aspect est devenu le vecteur permettant son inscription sur les listes du PCI. Aujourd'hui, le tahti ib, avec sa musique - rappelons que les joueurs ne les distinguent pas : ils sont parfaitement imbriqués -, figure dans l'inventaire culturel égyptien. Il est un patrimoine national légitime, chargé de valeurs et paré de vertus morales, valorisé par l'État au sein des maisons de la culture et des compétitions interrégionales ${ }^{41}$. Il est aussi une pratique «traditionnelle» qui, pour beaucoup de joueurs, s'oppose aux changements contemporains qu'implique la modernité. Considéré comme le dépositaire d'une histoire prestigieuse, le tahțib irait à l'encontre du bulldozer du monde contemporain globalisé. Les démarches liées à la pratique du tahțîb donnent un aperçu des constructions mémorielles actuellement à l'œuvre en Égypte, ainsi que la volonté de l'État de hiérarchiser certaines composantes culturelles égyptiennes bien spécifiques.

Pour justifier les propriétés traditionnelles du jeu, les discours mobilisent des éléments d'un passé pluriséculaire - la musique manifesterait, dans le jeu, un lien entre le ciel et la terre, selon Boulad, "symbolisé dans l'art égyptien de différentes manières [...] et [qui] fait partie des bases spirituelles de l'Égypte des Pharaons ${ }^{42} »-$ et les combinent avec d'autres aspects qui caractérisent le jeu. Le tahțīb est aujourd'hui une expression de "l'authenticité »: une authenticité de la pratique, en référence à son histoire admise qui l'inscrit dans une généalogie prestigieuse, et une authenticité masculine, en référence à l'idéal masculin qu'elle implique. Le tahțiib, avec ses chorégraphies et sa musique, constitue le théâtre de la "masculinité sa'idi ». Porteur d'un savoir-faire qui aurait traversé les âges et les époques, il garantit aussi la virilité de ses exécutants.

Pour permettre l'inscription au PCI, il a fallu envisager un développement de la pratique égyptienne. Le Modern Tahtib incarne ce développement. Il retire toutefois deux composantes de la pratique égyptienne: le jeu n'est plus ouvert qu'aux seuls hommes, mais aussi aux femmes. Quant à la musique, elle ne fait partie du jeu qu'en de rares occasions : le registre musical est écarté, faisant l'objet d'un travail de séparation qui hiérarchise les composantes du jeu. En vue de l'inscription du tahțiib au PCI, la

Transposition, 8 | 2019 
création du Modern Tahtib a permis de respecter les conditions de l'Unesco. Cette inscription, du fait des démarches patrimoniales qui lui sont liées, a toutefois un impact stylistique sur le jeu. Elle redéfinit un tahțiib où la musique n'est manifestement pas reconnue comme une composante indispensable, constitutive du jeu. L'exemple du jeu du bâton et de la place que la musique y occupe offre ainsi un exemple de patrimonialisation qui s'illustre par une tension entre les discours insistants sur la continuité d'une pratique pluriséculaire, efficace quand il s'agit de son inscription au $\mathrm{PCI}$, et des pratiques qui se transforment et se réadaptent à des contextes changeants.

\section{BIBLIOGRAPHIE}

ADELL Nicolas et POURCHER Yves (dir.), Transmettre, quel(s) patrimoine(s) ? Autour du Patrimoine Culturel Immatériel, Paris, Michel Houdiard, 2011.

BOULAD Adel, Modern Tahtib, bâton de combat égyptien, Budo, 2014.

CHIH Rachida, « Abu-l-Hajjaj al-Uqsuri, saint patron de Louqsor », Égypte/Monde arabe, Première série, $n^{\circ}$ 14, 1993, http://journals.openedition.org/ema/569, consulté le 11 septembre 2018. CHIFFOLEAU Sylvia et MADOEUf Anna (dir.), Les pèlerinages au Maghreb et au Moyen-Orient : espaces publics, espaces du public, Beyrouth, Ifpo, 2005.

CHIH Rachida, Le soufisme au quotidien : confréries d'Égypte au XX siècle, Arles-Paris, Sinbad-Actes Sud, 2000.

CoNNELl Raewyn, Masculinities, Cambridge, Polity Press, 1995.

EMERIT Sibylle, GUICHARD Hélène, JEAMMET Violaine,PERROT Sylvain, THOMAS Ariane, VENDRIES Christophe, VINCENT Alexandre et ZIEGLER Nele (dir.), Musiques ! Échos de l'Antiquité, Lens, Musée du Louvre-Lens, Gand, Snoeck Publishers, 2017.

GABRY-THIENPONT Séverine, " "Pharaonisme" et discours traditionnalistes : à la recherche du passé pour créer le présent », COIN-LONGERAY Sandrine (dir.), Instrumentalisation, mystification, réécritures des sources, Chemin de Tr@verse, 2014,p. 9-31.

GABRY-THIENPONT Séverine, «Du Caire à Nantes. Parcours et reformulations du zār, de ses musiques et de ses acteurs ", Cahiers d'ethnomusicologie, vol. 30, 2017, p. 137-153.

GUILLEBAUD Christine, «L'art de la multimodalité : musique, image et danse en Inde », Anthropologie et sociétés, vol. 38, $\mathrm{n}^{\circ} 1,2014$.

LAGRANGE Frédéric, Musiques d'Égypte, Paris-Arles, Cité de la musique-Actes Sud, 1999, p. 158.

LEGRAIN Georges, Louqsor sans les Pharaons, Paris, Vromant, 1914.

MAYEUR-JAOUEN Catherine, Histoire d'un pèlerinage légendaire en Islam : le mouled de Tantâ du XIII ${ }^{e}$ siècle à nos jours, Paris, Aubier, 2004.

MAYEUR-JAOUEN Catherine, Pèlerinages d'Égypte : histoire de la piété copte et musulmane, XVème-XXème siècles, Paris, EHESS, 2005. 
MILLER Catherine, «Between Myth and Reality: The Construction of the Sa'îdi Identity ", HOPKINS Nicholas and SAAD Reem (eds.), Upper Egypt, Identity and Change, Le Caire, AUC Press, 2004, p. 25-54. OULEBSIR Nabila et VOLAIT Mercedes (dir.), L'orientalisme architectural entre imaginaires et savoirs, Paris, Picard, 2009.

POUILLON François et VATIN Jean-Claude (dir.), Après l'orientalisme : l'Orient créé par l'Orient, Paris, IISMM-Karthala, 2011.

SAID Edward, L'orientalisme : l'orient créé par l'occident, Seuil, 2005 [1978], p. 15.

\section{NOTES}

1. Les mawālid musulmans sont des festivités célébrant l'«anniversaire de naissance » (traduction littérale du terme) du Prophète ou d'un wali (saint musulman; littéralement, proche de [Dieu]). Sur l'islam populaire égyptien et les festivités qui lui sont associées, voir MAYEURJAOUEN Catherine, Histoire d'un pèlerinage légendaire en Islam : le mouled de Tantâ du XIII ${ }^{e}$ siècle à nos jours, Paris, Aubier, 2004, ainsi que CHIH Rachida, Le soufisme au quotidien: confréries d'Égypte au $\mathrm{XX}^{e}$ siècle, Arles-Paris, Sinbad-Actes Sud, 2000.

2. Je remercie infiniment mon ami Tarek, compagnon de route de mes pérégrinations moulediennes en Haute Égypte depuis 2014, qui a eu la patience de m'accompagner à toutes ces festivités, me facilitant l'accès à un milieu très masculin.

3. El-Kom est un hameau du vaste village d'Al-Ba īrāt, situé sur la rive ouest de Louxor, village de Haute-Égypte où j'ai mené la majeure partie de mes enquêtes sur ce sujet.

4. Une occurrence de ce deuxième sens se trouve dans un film égyptien de 1981, Fetewat Boulaq, réalisé par Yahiya El-'Alami et inspiré d'un roman de Naguib Mahfouz, mettant en scène des gangs dans le quartier cairote de Boulaq.

5. LAGRANGE Frédéric, Musiques d'Égypte, Paris-Arles, Cité de la musique-Actes Sud, 1999, p. 158.

6. Extrait vidéo de l'auteure à consulter à l'adresse suivante : archive.org/details/Tahtib. Notons aussi l'existence du documentaire Al bab, réalisé par Abdul Fattah Kamal, qui présente la pratique du tahțīb à travers une figure notable du jeu, Medhat Fawzy (à présent décédé), en lien avec le metteur en scène et fondateur de la troupe théâtrale El-Warsha Hasan El Geretly, et les élèves du centre de Mallawi (ville située à environ $300 \mathrm{~km}$ au sud du Caire), seule école de tahțîb existant en Égypte, financée par El-Warsha.

7. Sur l'organisation spatiale des mawālid en Égypte, se référer à MAYEUR-JAOUEN Catherine, Pèlerinages d'Égypte : histoire de la piété copte et musulmane, XVème-XXème siècles, Paris, EHESS, 2005, et à CHIFFOLEAU Sylvia et MADOEUF Anna (dir.), Les pèlerinages au Maghreb et au Moyen-Orient : espaces publics, espaces du public, Beyrouth, Ifpo, 2005.

8. Ces manifestations, bien souvent organisées lors de festivités religieuses, sont systématiquement précédées de la récitation de la première sourate, visant à garantir le bon déroulement du jeu, sous de saints auspices.

9. En Égypte, excepté dans les quelques conservatoires du nord du pays où les femmes apprennent à jouer des instruments de musique mélodiques pour aborder des répertoires dits "savants", la répartition genrée du jeu instrumental est extrêmement claire et balisée : les femmes chantent et jouent des percussions, mais pas d'instruments à vent ni à corde. Je n'ai jamais rencontré de femmes jouant du mizmār. Cette considération particulière d'un jeu instrumental autorisé à la gent féminine n'est sans doute pas figée dans le marbre: un joueur professionnel d'arghoul et de kawala du Caire me racontait qu'il enseignait ces instruments à l'une de ses filles âgée de 8 ans, à sa demande. Le phénomène demeure néanmoins marginal pour l'instant. 
10. Guillebaud Christine, "L'art de la multimodalité: musique, image et danse en Inde", Anthropologie et sociétés, vol. 38, $\mathrm{n}^{\circ} 1,2014$, p. 25-45. Citation p. 40.

11. Ibid., p. 26.

12. Ibid., p. 30.

13. D'autres pratiques culturelles égyptiennes ne bénéficient pas de ce statut : c'est par exemple le cas du rituel de possession zār, qui a mauvaise presse et n'est pas reconnu comme patrimoine égyptien par les autorités étatiques - alors qu'il l'est par des entrepreneurs culturels privés au Caire (GABRY-THIENPONT Séverine, «Du Caire à Nantes. Parcours et reformulations du zār, de ses musiques et de ses acteurs ", Cahiers d'ethnomusicologie, vol. 30, 2017, p. 137-153).

14. MILLER Catherine, "Between Myth and Reality: The Construction of the Sa'îdi Identity", HOPKINS Nicholas and SAAD Reem (eds.), Upper Egypt, Identity and Change, Le Caire, AUC Press, 2004, p. 25-54.

15. CONNELl Raewyn, Masculinities, Cambridge, Polity Press, 1995.

16. L'un de mes interlocuteurs particulièrement rusé et habile était par exemple désigné par ses compagnons de jeu comme « le renard».

17. BoulaD Adel, Modern Tahtib, bâton de combat égyptien, Budo, 2014, p. 14.

18. ADELL Nicolas et POURCHER Yves (dir.), Transmettre, quel(s) patrimoine(s)? Autour du Patrimoine Culturel Immatériel, Paris, Michel Houdiard, 2011, p. 9.

19. GABRY-THIENPONT Séverine, " "Pharaonisme" et discours traditionnalistes : à la recherche du passé pour créer le présent», COIN-LONGERAY Sandrine (dir.), Instrumentalisation, mystification, réécritures des sources, Chemin de Tr@verse, 2014, p. 9-31.

20. Nous pensons ici aux travaux de Hans Hickmann, René Ménard et Ilona Borsai, consacrés aux chants de la liturgie copte.

21. SAID Edward, L'orientalisme : l'orient créé par l'occident, Seuil, 2005 [1978], p. 15.

22. Concernant les clichés répandus autour des musiques de l'Antiquité, on se réfèrera à l'excellent catalogue de l'exposition « Musiques! Échos de l'Antiquité »: EMERIT Sibylle, GUICHARD Hélène, JEAMMET Violaine,PERROT Sylvain, THOMAS Ariane, VENDRIES Christophe,vinCENT Alexandre et ZIEGLER Nele (dir.), Musiques! Échos de l'Antiquité, Lens, Musée du Louvre-Lens, Gand, Snoeck Publishers, 2017, plus particulièrement aux pages 116 à 127.

23. CHIH Rachida, "Abu-l-Hajjaj al-Uqsuri, saint patron de Louqsor», Égypte/Monde arabe, Première série, $\mathrm{n}^{\circ}$ 14, 1993, journals.openedition.org/ema/569 (consulté le 11 septembre 2018).

24. LEGRAIN Georges, Louqsor sans les Pharaons, Paris, Vromant, 1914, p. 87, cité par CHIH, op.cit.

25. Il est possible de visionner ce film à l'adresse suivante: videotheque.cnrs.fr/index.php? urlaction=doc\&id_doc=499\&rang=1\# (consulté le 11 septembre 2018).

26. POUILLON François et VATIN Jean-Claude (dir.), Après l'orientalisme : l'Orient créé par l'Orient, Paris, IISMM-Karthala, 2011.

27. OULEBSIR Nabila et volAIT Mercedes, «Introduction », OULEBSIR Nabila et volAIT Mercedes (dir.), L'orientalisme architectural entre imaginaires et savoirs, Paris, Picard, 2009, p. 7-10.

28. $\quad$ youtube.com/watch?v=quYxwYqYux4\&list=PL11giPLtNu3Wbx93-81Pq6BbPAIjExPL\&index=25 (consulté le 25 juin 2018).

29. Notons que cette manière, artistique, de représenter le tahtīib a fait des émules : il existe en effet à l'heure actuelle une troupe, menée par l'Égyptien Hasan El-Geretly, spécialisée dans les créations chorégraphiques autour du tahțiib. La troupe a été présentée au public français en mars 2018 au Musée du Quai Branly.

30. Convention pour la sauvegarde du patrimoine culturel immatériel, comité intergouvernemental de sauvegarde du patrimoine culturel immatériel, Onzième session, Addis-Abeba, Éthiopie, 28 novembre - 2 décembre 2016, Décisions, p. 34-36, paru le 22 décembre 2016 et téléchargeable en ligne à l'adresse suivante : ich.unesco.org/en/11com (consulté le 25 juin 2018). 
31. Ayman Khoury a longtemps travaillé à CultNat, The Center for Documentation of Cultural and Natural Heritage, centre de recherche cairote rattaché à la Bibliotheca Alexandrina et soutenu par le Ministère de la communication et des technologies de l'information. Photographe de formation, il a réalisé dans le cadre de ce poste un important travail de documentation des pratiques égyptiennes, surtout musicales, dont toutes les archives, difficiles d'accès, sont conservées à CultNat. Pour la constitution du dossier mis en œuvre par Adel Boulad, A. Khoury avait accepté d'enquêter et de réaliser un film de présentation du jeu, consultable depuis 2016 sur la chaîne YouTube de l'Unesco: youtube.com/watch?v=QkbEI7co0y4\&t=47s (consulté le 6 septembre 2018).

Qu'il soit ici chaleureusement remercié pour m'avoir permis d'assister à son entretien avec Mahmūd Manșūr, l'un des joueurs interrogés figurant dans le petit film susmentionné, en 2013, et avant ça, pour les nombreuses clés de compréhension qu'il m'a données lors de mes recherches en Égypte, avant que les événements de 2011 ne l'amènent à quitter le pays.

32. Pour consulter un exemple de cette pratique, voir par exemple: youtube.com/watch? v=GTHIIxKi6js (consulté le 6 septembre 2018).

33. tahtib.com/tahtib_project (consulté le 6 septembre 2018).

34. La question particulière de la transmission des patrimoines et sur les problématiques qu'elle sous-tend a été largement traitée dans ADELL et POURCHER, Transmettre, quel(s) patrimoine(s)?

35. tahtib.com/tahtib_project (consulté le 6 septembre 2018).

36. Instrument à percussion à membrane simple.

37. BOULAD, Modern Tahtib, p. 230-235. L'analogie entre passé et présent est constante : «Le mot "souverain" s'écrit avec le dessin [hiéroglyphique] du bâton. Tout homme peut s'en emparer pour exprimer son rôle et son autorité. De nos jours, en Haute-Égypte, les villageois en témoignent fièrement ». Ibid., p. 231. Il existe donc des scènes qui représenteraient des lutteurs avec leurs instructeurs. Il s'agit de scènes d'entrainement. Les individus sont munis de bâtons longs, ce qui est différent d'autres scènes d'entrainement avec des bâtons courts, dont les codes apparemment diffèrent. Ibid., p. 232.

38. Ibid., p. 220.

39. Id.

40. tahtib.com/media|articles|Tahtib\%20inscrit\%20à\%20l'UNESCO (consulté le 6 septembre 2018).

41. Les problématiques patrimoniales en Égypte épousent différentes formes et relèvent de différents acteurs. Il est ainsi nécessaire de différencier ce qui est de l'ordre de l'état et ce qui est de l'ordre de l'entreprenariat culturel privé, qui se développe particulièrement au Caire depuis les années 1990. Les deux sont extrêmement cloisonnés et dévoilent des choix divergents de politiques d'archivage, de modes de collecte et de valorisation patrimoniale : tant de choix qui révèlent un jeu complexe de pouvoir, de censure, et de représentation nationale de ce que doit être la culture.

42. BOULAD, Modern Tahtib, p. 220.

\section{RÉSUMÉS}

Classé depuis novembre 2016 au PCI, le tahțiib est une pratique égyptienne qui se joue particulièrement pendant les fêtes de mariage et les pèlerinages (mawlid). Il s'agit d'un jeu aux règles strictes, exclusivement masculin: deux hommes munis d'un bâton s'affrontent, en un 
enchaînement chorégraphique aux figures définies. La musique y occupe une place centrale : mizmār et țabla baladi accompagnent les joueurs, les encouragent, les retiennent. Comme nombre de pratiques rurales, le tahtịib est considéré tant par ses acteurs que par les spectateurs comme une tradition ancestrale, dont l'origine remonterait à l'Égypte pharaonique. Après une présentation de la pratique du tahțî̉ dans l'Égypte du XXI ${ }^{\mathrm{e}}$ siècle, je propose d'analyser les discours à teneur pharaoniste, les processus de patrimonialisation qui en découlent, ainsi que les récents développements qui ont mené à la création d'un Modern Tahtib par Adel Boulad. Cette réflexion permettra de questionner les constructions mémorielles actuellement à l'œuvre en Égypte, leur réception à l'étranger - en l'occurrence, en France -, et la manière dont elles ont permis l'inscription du tahțîb et de sa musique au PCI.

Inscribed on the ICH list since 2016, tahțib is an Egyptian practice typically performed at wedding celebrations and pilgrimages (mawlid). It is an exclusively male martial art with strict rules, in which two men, each armed with a stick, perform a combat in a choreographed series of defined figures. Music is central to tahtîib: the players are accompanied-encouraged and restrained-by the playing of mizmār and tabla baladi. Like many rural practices, tahțīb is considered by both its participants and spectators to be an ancestral tradition tracing back to Egypt's Pharaonic era. After presenting the practice of tahtib in twenty-first century Egypt, I will examine elements of the Pharaonist discourse, the resulting heritagisation processes, and the recent developments that led to Adel Boulad's creation of Modern Tahtib. This analysis will focus on the memory constructions currently at play in Egypt, their reception abroad-in this case, in France-and how they allowed tahtîib and its music to be inscribed on the ICH list.

\section{INDEX}

Mots-clés : tahtib, Égypte, masculinité, Unesco, patrimonialisation, jeu

Keywords : tahtib, Egypt, masculinity, Unesco, heritagisation, martial art

\section{AUTEUR}

\section{SÉVERINE GABRY-THIENPONT}

Séverine Gabry-Thienpont est ethnomusicologue, chargée de recherche à l'Institut d'ethnologie méditerranéenne, européenne et comparative (Idemec, CNRS). De 2013 à 2017, elle a mené au sein de l'IFAO (Le Caire) un projet de recherche consacré aux musiques des manifestations de piété populaire en Égypte. S. Gabry-Thienpont oriente désormais ses recherches sur la fabrique des musiques égyptiennes en tant que fait esthétique, historique et religieux, à l'aune des procédés d'amplification sonore auxquels elles sont soumises. Elle propose ainsi d'écrire une histoire de la modernité sonore de l'Égypte, depuis les premiers enregistrements réalisés dans un but patrimonial au début $\mathrm{du} \mathrm{XX}^{\mathrm{e}}$ siècle, jusqu'aux compositions liées aux pratiques actuelles du « Do It Yourself ». 